\title{
Satisfaction and quality of life in patients with
} symptomatic gastroenteropancreatic neuroendocrine tumours treated with lanreotide Autogel in South Africa

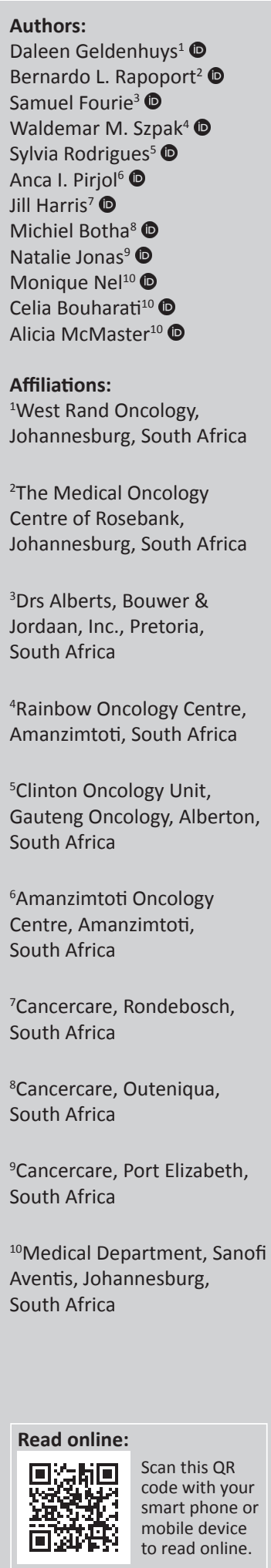

Background: Neuroendocrine tumours are known to impact patients' quality of life because of the symptoms caused by hypersecretion of serotonin and other peptides, in particular diarrhoea and flushing.

Aim: The Q-SYMTU study was a prospective, observational registry that included 24 symptomatic patients with gastroenteropancreatic neuroendocrine tumours.

Setting: Multiple oncology practices in South Africa.

Method: Patients' level of satisfaction was evaluated for a 6-month period from initiation of treatment with lanreotide Autogel.

Results: The number of patients who had greater than $50 \%$ self-reported reduction in daily episodes of diarrhoea and flushing were $67 \%$ and $80 \%$, respectively, over a 6-month period.

Conclusion: Treatment with lanreotide Autogel was generally well tolerated, as demonstrated by low occurrence of Grade 3 and Grade 4 adverse events (AEs). None of the Grade 4 AEs were related to the study treatment. No Grade 5 AEs were reported.

Keywords: lanreotide Autogel; somatostatin analogues; neuroendocrine tumours; gastroenteropancreatic neuroendocrine tumours; oncology.

\section{Introduction}

Gastroenteropancreatic neuroendocrine tumours (GEP-NETs) are rare neoplasms arising from the endocrine cells of the gastrointestinal (GI) tract and the pancreas that secrete serotonin and other peptides. ${ }^{1}$ This secretion causes a complex clinical spectrum, including carcinoid syndrome (e.g. diarrhoea and skin flushing), which affects the patients' health-related quality of life (HR-QoL).

Although considered as rare, the incidence of GEP-NETs has increased to 3 cases per 100000 people per year, with a slight predominance in women. ${ }^{2}$ Prevalence data for South Africa is lacking.

The only potential curative treatment is surgery or cytoreduction when the tumour is localised; however, more than $80 \%$ of patients present with metastases. Other options include radiological intervention, chemotherapy and somatostatin analogues such as lanreotide Autogel. Somatostatin analogues can control hypersecretion of peptides by NETs that express somatostatin receptors. In somatostatin receptor 2 (sst2) or somatostatin receptor 5 (sst5) positive tumours, clinical symptoms related to hypersecretion can be controlled by long-term administration of one of the currently available somatostatin analogues. ${ }^{3}$

Self-reported QoL measurements are increasingly being used as end points in oncology studies. ${ }^{2}$ Improvement in HR-QoL is one of the potential benefits that are considered by the Food and Drug Administration (FDA) for the approval of new anti-cancer drugs. ${ }^{4}$ In this study, the validated QLQ-GINET21 questionnaire was used to evaluate QoL at baseline (before lanreotide Autogel

Project research number: LANREL07484

Corresponding author: Monique Nel, monique.nel@sanofi.com

Dates: Received: 15 June 2020 | Accepted: 01 July 2020 | Published: 01 Oct. 2020

How to cite this article: Geldenhuys D, Rapoport BL, Fourie S, et al. Satisfaction and quality of life in patients with symptomatic gastroenteropancreatic neuroendocrine tumours treated with lanreotide Autogel in South Africa. S. Afr. j. oncol. 2020;4(0), a138. https:// doi.org/10.4102/sajo.v4i0.138

Copyright: @ 2020. The Authors. Licensee: AOSIS. This work is licensed under the Creative Commons Attribution License. 
was initiated), at 3 months after starting lanreotide Autogel treatment and again at 6 months post-initiation of therapy.

This is the first national, multi-centre, prospective product registry to measure patient satisfaction with control of diarrhoea and/or flushing, QoL, safety and tolerability during a 6-month period of therapy with lanreotide Autogel in patients with symptomatic GEP-NETs in South Africa.

\section{Methodology and objectives Patients}

Q-SYMTU (LANREL07484) was a multi-centre, prospective observational cohort study sponsored by Sanofi South Africa. The study protocol was approved by the independent institutional ethics committee, and a written informed consent was obtained from each patients before study entry, in accordance with the Declaration of Helsinki. ${ }^{5}$ Adult patients ( $\geq 18$ years) were enrolled in the registry from 10 oncology practices. Study sites were selected based on the number of symptomatic GEP-NET patients treated per annum at the site. Patients who had participated in any clinical trial and/or treatment with any investigational drug within 30 days were excluded from participation in this registry, as well as patients who were pregnant, lactating or who had unresolved National Cancer Institute Common Terminology Criteria for Adverse Events (NCI CTC < 1) or unstable toxicity from any prior anti-cancer therapy at the time of enrolment.

\section{Treatment}

All patients enrolled in the study were treated with lanreotide Autogel. All participating physicians prescribed lanreotide Autogel independently from the study and were therefore guided by the registered prescribing information, their usual treatment protocols and practices, international treatment guidelines and ethical considerations. The design of the study reflected real-life management of patients with proven diagnosis of GEP-NET with uncontrolled hormone-related symptoms (diarrhoea and/or flushing). Patients were monitored at baseline, 3 months post-initiation of treatment with lanreotide Autogel and 6 months post-initiation of treatment. The patient or physician could decide to withdraw from the treatment at any time for any reason.

\section{Efficacy and safety assessments}

The primary end points of the study included evaluation of patients' level of satisfaction with control of diarrhoea and/ or flushing when treated with lanreotide Autogel over a 6-month period as well as the evaluation of symptomatic response of diarrhoea and/or flushing over a 6-month period. Secondary end points included evaluation of QoL, exposure to treatment and safety.

\section{Statistical methodology}

Based on the large pooled analysis completed by Modlin et al., ${ }^{6}$ it was estimated that a minimum sample size of
29 patients will provide $80 \%$ power to detect a statistically significant clinical response to lanreotide Autogel at the 0.05 significance level, allowing for a $20 \%$ attrition. Stata version 14.1 (StataCorp, College Station, TX, USA) was used to generate this sample size.

These calculations were performed according to assumptions based on previous studies and the study design:

- Single cohort non-interventional study with two occasions (baseline and 6-month follow-up).

- Variable used for sample size estimation: symptomatic score (assumed as a continuous variable).

- Assumed effect size of $>50 \%$ (so effect size was set as $0.55)$, assuming that the symptomatic score prior to intervention (i.e. at baseline) was equal to 6 (standard deviation [s.d.], 2.3) and that there is a $50 \%$ reduction to a mean of 3 .

- Alpha was set at 0.05 (5\% chance of having a false positive result).

The statistical calculation for a one-sided test gives a minimum number of 24 patients to allow for a $20 \%$ attrition; an additional 5 patients results in an attrition rate of $20.69 \%$, yielding a sample size of 29 .

Data from all the participating practices in South Africa were combined and treated as one dataset for the purposes of the analysis. The statistical analysis of the survey was of a descriptive nature, where continuous variables were summarised by mean, median, standard deviation and minimum and maximum values and discrete variables were summarised by frequencies and percentages. All analyses were carried out using Stata version 15.1.

\section{Ethical consideration}

Ethical approval to conduct the study was obtained from the independent institutional ethics committee (reference no. PHARMA-ETHICS - 150411304).

\section{Results}

\section{Trial population}

Between June 2015 and June 2018, 24 patients were enrolled in the Q-SYMTU (LANREL07484) study, and they all received lanreotide Autogel. In total, 16 patients $(66.7 \%)$ completed the study, whereas $8(33.3 \%$ ) left the study prematurely ( 3 for disease progression, 1 for death, 1 for withdrawal, 1 following the physician's decision, 1 for reimbursement being declined and 1 for treatment being stopped).

The mean age of the patients was 63.5 years old and $58.3 \%$ of them were women. Most of the patients were white people of European descent (18 patients; 75\%). At baseline, 79.2\% of the patients presented with a relevant medical condition, the most frequent being metabolism and nutrition disorders (32.1\%). In addition, $20.8 \%$ of the patients had complications related to the carcinoid syndrome and/or NET, the main one 
being skin complications (other than flushing). Three-quarters of the patients were receiving anticancer and NET-related therapy at baseline, with octreotide being the most frequently used treatment $(77.8 \%)$. Anti-diarrhoeal treatment and symptomatic treatment for flushing was used by 10 patients $(41.7 \%)$ in the 2 months preceding enrolment in the study (Table 1).

\section{Diarrhoea and flushing}

To evaluate the improvement or stabilisation in level of satisfaction of diarrhoea and/or flushing control, patients

TABLE 1: Patient characteristics at baseline.

\begin{tabular}{|c|c|c|c|}
\hline \multirow[t]{2}{*}{ Characteristics } & \multicolumn{3}{|c|}{ Value } \\
\hline & Years & $n$ & $\%$ \\
\hline Age at baseline & $63.5 \pm 10.4$ & - & - \\
\hline \multicolumn{4}{|l|}{ Gender } \\
\hline Male & - & 10 & 41.7 \\
\hline Female & - & 14 & 58.3 \\
\hline \multicolumn{4}{|l|}{ ECOG performance status } \\
\hline 0 & - & 9 & 37.5 \\
\hline 1 & - & 15 & 62.5 \\
\hline \multicolumn{4}{|l|}{ Site of primary tumour } \\
\hline Small bowel & - & 14 & 58.3 \\
\hline Colon & - & 3 & 12.5 \\
\hline Pancreas & - & 2 & 8.3 \\
\hline Unknown & - & 2 & 8.3 \\
\hline Appendix & - & 0 & 0 \\
\hline Other & - & 3 & 12.5 \\
\hline Gastric & - & 1 & 5.6 \\
\hline Possibly pancreas & - & 1 & 5.6 \\
\hline Rectum & - & 1 & 5.6 \\
\hline \multicolumn{4}{|l|}{ Hepatic tumour volume } \\
\hline $0 \%$ & - & 7 & 29.2 \\
\hline$>0 \%$ to $\leq 10 \%$ & - & 5 & 20.8 \\
\hline$>25 \%$ to $\leq 50 \%$ & - & 1 & 4.2 \\
\hline$>50 \%$ & - & 4 & 16.7 \\
\hline Not available & - & 7 & 29.2 \\
\hline \multicolumn{4}{|c|}{ Anti-cancer/NET treatment at baseline } \\
\hline No & - & 6 & 25 \\
\hline Yes & - & 18 & 75 \\
\hline Octreotide (Sandostatin) & - & 15 & 83.3 \\
\hline Intron & - & 1 & 5.6 \\
\hline Lu-177 dotatate & - & 1 & 5.6 \\
\hline MIBG treatment & - & 1 & 5.6 \\
\hline \multicolumn{4}{|l|}{ Lab results, $\mathrm{CgA}(n=25) \uparrow$} \\
\hline Mean & 1154.9 & - & - \\
\hline \pm s.d. & \pm 1965.3 & - & - \\
\hline Median & 229 & - & - \\
\hline IQR & $98.9-210$ & - & - \\
\hline
\end{tabular}

CgA, Chromogranin A; ECOG, Eastern Cooperative Oncology Group; IQR, interquartile range; MIBG, Metaoidobenzylguanidine; NET, neuroendocrine tumour; s.d., standard deviation.

Total population, $N=24(100 \%)$.

$\dagger$, One patient had two baseline results for CgA. completed a satisfaction survey utilising a Likert scale. A total of 17 patients completed this satisfaction survey at both baseline and the 3-month visit, and 14 patients completed the satisfaction survey at both baseline and the 6-month visit. In all, 13 patients $(76.5 \%)$ reported improvement and/or stabilisation in their level of satisfaction with diarrhoea control from baseline to the 3-month visit and 11 patients $(78.6 \%)$ reported improvement/stabilisation in diarrhoea from baseline to the 6-month visit. When asked about their flushing control, 16 (94.1\%) patients showed improvement and/or stabilisation in level of satisfaction from baseline to 3 months and all the patients (100\%) reported improvement or stabilisation from baseline to the 6-month follow-up.

Frequency of diarrhoea and flushing was sufficiently completed for 12 patients at the baseline and 3-month visit for evaluation of response to treatment. This information was evaluable for 9 patients from baseline to the 6 month-visit for diarrhoea and 10 patients for flushing. Majority of patients experienced at least $50 \%$ improvement in the number of average daily episodes of diarrhoea $(9 / 12 ; 75.0 \%)$ and flushing $(7 / 12 ; 58.3 \%)$ during the first 3 months. Between baseline and 6 months, 6 patients $(66.7 \%)$ reported at least $50 \%$ improvement in the number of average daily episodes of diarrhoea and 8 (80\%) patients experienced at least 50\% improvement in the number of average daily episodes of flushing.

At enrolment, 16 patients reported daily episodes of diarrhoea. Five of twenty-four patients enrolled did not report an episode of diarrhoea at any visit they attended and are not included in Table 2. Three of twenty-four diarrhoea episodes were not included in the summary statistics as they were reported as unknown.

In patients who reported at least one episode of diarrhoea, there was a statistically significant reduction in the mean and median number of daily episodes of diarrhoea from baseline to the 3-month follow-up visit ( $p=0.019$ and $p=0.006$ ); however, this significance was not observed between baseline and the 6-month follow-up visit, or between the 3-month and 6-month follow-up visits (Table 2).

At enrolment, only 18 patients reported with the number of daily episodes of flushing (mean [ \pm s.d.]) being $1.8( \pm 2.2)$ and the median (interquartile range [IQR]) 1.0 (0.1-3.0). In patients who reported at least one episode of flushing, there was a reduction in the median number of episodes of flushing from 1.0 per day (baseline) to 0.0 median episodes per day at month 6 (Table 3).

TABLE 2: Change in episodes of diarrhoea during 6 months of follow-up.

\begin{tabular}{|c|c|c|c|c|c|c|c|c|c|}
\hline \multirow[t]{3}{*}{ Episodes of diarrhoea } & \multicolumn{6}{|c|}{ In patients with at least one episode of diarrhoea } & \multicolumn{3}{|c|}{$p$-value for the difference in diarrhoea episodes over visits } \\
\hline & \multicolumn{2}{|c|}{$\begin{array}{c}\text { Baseline } \\
(n=16)\end{array}$} & \multicolumn{2}{|c|}{$\begin{array}{l}\text { 3-month visit } \\
\quad(n=16)\end{array}$} & \multicolumn{2}{|c|}{$\begin{array}{l}\text { 6-month visit } \\
\quad(n=12)\end{array}$} & \multirow[t]{2}{*}{$\begin{array}{l}\text { Baseline to } \\
3 \text { months }\end{array}$} & \multirow[t]{2}{*}{$\begin{array}{l}\text { Baseline to } \\
6 \text { months }\end{array}$} & \multirow[t]{2}{*}{ 3-6 months } \\
\hline & $n$ & $\%$ & $n$ & $\%$ & $n$ & $\%$ & & & \\
\hline Mean (s.d.) episodes of diarrhoea per day & 3.8 & 4.1 & 1.2 & 3 & 1.8 & 3.3 & 0.019 & 0.134 & 0.451 \\
\hline Median (IQR) episodes of diarrhoea per day & 2.5 & $0.5-6.5$ & 0.3 & $0.0-0.8$ & 0.1 & $0.0-2.0$ & 0.006 & 0.083 & 0.579 \\
\hline
\end{tabular}

s.d., standard deviation; IQR, interquartile range. 
TABLE 3: Change in episodes of flushing during 6 months of follow-up.

\begin{tabular}{|c|c|c|c|c|c|c|c|c|c|c|c|c|}
\hline \multirow[t]{3}{*}{ Episodes of flushing } & \multicolumn{9}{|c|}{ In patients with at least one episode of flushing } & \multicolumn{3}{|c|}{$p$-value for the difference in flushing episodes over visits } \\
\hline & \multicolumn{3}{|c|}{ Baseline $(n=18)$} & \multicolumn{3}{|c|}{ 3-month visit $(n=17)$} & \multicolumn{3}{|c|}{ 6-month visit $(n=13)$} & \multirow{2}{*}{$\begin{array}{l}\text { Baseline to } \\
3 \text { months }\end{array}$} & \multirow{2}{*}{$\begin{array}{c}\text { Baseline to } \\
6 \text { months }\end{array}$} & \multirow[t]{2}{*}{ 3-6 months } \\
\hline & $n$ & \pm s.d. & IQR & $n$ & \pm s.d. & IQR & $n$ & \pm s.d. & IQR & & & \\
\hline Mean episodes of flushing per day & 1.8 & \pm 2.2 & - & 1.2 & \pm 1.7 & - & 0.7 & \pm 1.2 & - & 0.551 & 0.202 & 0.172 \\
\hline Median episodes of flushing per day & 1.0 & - & $0.1-3.0$ & 0.1 & - & $0.1-2.0$ & 0.0 & - & $0.0-0.3$ & 0.255 & 0.169 & 0.318 \\
\hline
\end{tabular}

s.d., standard deviation; IQR, interquartile range.

TABLE 4: Change in QoL from baseline.

\begin{tabular}{|c|c|c|c|c|c|c|c|c|c|c|c|}
\hline \multirow[t]{2}{*}{ Variable } & \multicolumn{3}{|c|}{ Baseline } & \multicolumn{4}{|c|}{ 3-month visit } & \multicolumn{4}{|c|}{ 6-month visit } \\
\hline & $N$ & Mean & $\pm s . d$ & $N$ & Mean & $P$ & \pm s.d. & $N$ & Mean & $P$ & $\pm s . d$ \\
\hline \multicolumn{12}{|l|}{ QLQ-C30 } \\
\hline Total number of patients & $23 \dagger$ & - & & 19 & - & - & - & 12 & - & - & - \\
\hline Global health status & 21 & 50 & \pm 22.3 & 19 & 54.4 & 0.513 & \pm 19.1 & 12 & 73.6 & 0.005 & \pm 19.4 \\
\hline Functional status & 21 & 68.4 & \pm 24.7 & 19 & 68.6 & 0.977 & \pm 17.5 & 12 & 75.2 & 0.431 & \pm 20.7 \\
\hline Symptom status & 21 & 30.0 & \pm 23.4 & 19 & 33.2 & 0.665 & \pm 17.5 & 12 & 27.6 & 0.759 & \pm 19.2 \\
\hline \multicolumn{12}{|l|}{ GI.NET21 } \\
\hline Endocrine status & 23 & 39.0 & \pm 29.7 & 19 & 27.9 & 0.190 & \pm 23.0 & 12 & 8.3 & 0.002 & \pm 14.9 \\
\hline GI scale & 23 & 44.9 & \pm 27.9 & 19 & 29.6 & 0.049 & \pm 18.9 & 12 & 22.1 & 0.019 & \pm 22.1 \\
\hline Treatment scale & 6 & 16.5 & \pm 18.1 & 18 & 9.2 & 0.339 & \pm 15.2 & 10 & 9.9 & 0.458 & \pm 15.9 \\
\hline Social function scale & 23 & 46.3 & \pm 26.3 & 19 & 31.4 & 0.052 & \pm 20.8 & 12 & 33.3 & 0.187 & \pm 28.6 \\
\hline Disease-related worries scale & 13 & 46.2 & \pm 29.2 & 14 & 35.7 & 0.396 & \pm 33.3 & 9 & 44.6 & 0.911 & \pm 37.4 \\
\hline \multicolumn{12}{|c|}{ Item scores for muscle pain, sexual function, information or communication and body image } \\
\hline Muscle or bone pain & 23 & 45 & \pm 35.8 & 19 & 40.4 & 0.659 & \pm 30.7 & 12 & 36.1 & 0.454 & \pm 26.6 \\
\hline $\begin{array}{l}\text { Information or communication } \\
\text { function }\end{array}$ & 22 & 13.6 & \pm 22.2 & 19 & 8.8 & 0.491 & \pm 21.9 & 12 & 5.5 & 0.256 & \pm 12.8 \\
\hline Body image & 23 & 39.1 & \pm 41.1 & 19 & 33.3 & 0.652 & \pm 41.6 & 12 & 19.4 & 0.179 & \pm 38.8 \\
\hline
\end{tabular}

$\dagger$, One patient did not have a result for the baseline QoL questionnaire.

$\mathrm{Gl}$, gastrointestinal; s.d., standard deviation; QoL, quality of life.

\section{Quality of life}

The investigation of QoL in GEP-NET patients is a relatively new practice for which a disease-specific and validated questionnaire, the European Organization for Research and Treatment of Cancer (EORTC) QLQ-C304/7/QLQ-GI. NET215, is now being utilised in NET studies. The QLQ-C30 incorporates nine multi-item scales: five functional scales (physical, role, cognitive, emotional and social), three symptom scales (fatigue, pain, and nausea and vomiting) and a global health and QoL scale. Several single-item symptom measures are also included. The GI.NET21 module consists of 21 questions, which include assessment of disease symptoms, safety of treatment, body image, disease-related concerns, social functioning, communication and sexuality.

The reported $p$-values were the differences between baseline versus the 3-month visit and baseline versus the 6-month visit (Table 4).

\section{Quality of life using the QLQ-C30}

Scores on the QLQ-C30 are linearly transformed on a 1-100 scale, with higher scores representing an increased level of function and a higher level of symptomatology. ${ }^{8}$ As defined by Osaba et al.: ${ }^{9}$

[M]ean changes in HRQoL scores over time of 5 to 10 points are considered as 'small', 10 to 20 points as 'moderate', and more than 20 points as 'large' with regards to clinical relevance. (p. 139)
An increase in global health status scale score from baseline to the 3-month visit and from baseline to the 6-month visit was observed. From baseline to the 6-month visit, the increase of more than 20 points can be regarded as clinically relevant.

A slight non-significant improvement in functional status scale scores between baseline and the 6-month visit was observed.

A slight increase in symptom scale scores from baseline to the 3-month visit and a slight decrease between baseline and the 6-month visit were observed.

\section{Quality of life using GI.NET 21}

The scores are linearly transformed with lower scores representing an improved outcome from the various measurements between baseline and the 3-month visit and between baseline and the 6-month-visit.

A decrease was observed in endocrine-related symptom scale scores from baseline to the 3-month visit and from baseline to the 6-month visit. The results between baseline and 6-month showed a clinically relevant reduction of greater than 20 points.

A decrease in the scores of the gastrointestinal symptom scale, treatment-related problems scale, social function scale and disease-related worries scale was observed between baseline and the 3-month visit and between baseline and the 6-month visit. 
Item scores for muscle pain symptom, sexual function, information or communication and body image

The scores for the daily living activities are linearly transformed with lower scores representing an improved outcome for the various measurements.

A decrease of the scores was observed on the muscle or bone pain symptom scale, the information or communication function and in body image from baseline to the 3-month visit and from baseline to the 6-month visit.

With regards to the sexual dysfunction scale scores, an increase was observed between baseline and 3 months, and no change occurred between baseline and the 6-month visit.

\section{Treatment exposure}

Treatment with lanreotide Autogel was initiated at a median (IQR) dose of $90(60-120) \mathrm{mg} ; 8 / 24(33 \%)$ of the patients started treatment with a $120-\mathrm{mg}$ dose.

More than half of the patients received up to $6(29.2 \%)$ and $7(37.5 \%)$ treatment cycles.

No doses were reported as omitted. There were five dose delays (two because of vacation, one because of surgery, one because of an endoscopy, one because of stock supply), five patients who required a dose increase (three for disease/ symptom control, one for disease progression and one for reimbursement approved for a higher dose) and two patients

TABLE 5: Adverse events and serious adverse events by primary system organ class (SOC).

\begin{tabular}{|c|c|c|c|c|c|c|c|c|}
\hline \multirow[t]{2}{*}{ Primary system organ class } & \multicolumn{2}{|c|}{ All grades } & \multicolumn{2}{|c|}{ Grade 3} & \multicolumn{2}{|c|}{ Grade 4} & \multicolumn{2}{|c|}{ Grade 5} \\
\hline & $n$ & $\%$ & $n$ & $\%$ & $n$ & $\%$ & $n$ & $\%$ \\
\hline All systems & 33 & 100.0 & 6 & 18.2 & 3 & 9.1 & 0 & 0.0 \\
\hline Gastrointestinal disorders & 8 & 24.2 & 1 & 16.7 & 1 & 33.3 & 0 & 0.0 \\
\hline Diarrhoea & 3 & 9.1 & 1 & 16.7 & 0 & 0.0 & 0 & 0.0 \\
\hline Abdominal discomfort & 1 & 3.0 & 0 & 0.0 & 0 & 0.0 & 0 & 0.0 \\
\hline Abdominal pain & 1 & 3.0 & 0 & 0.0 & 0 & 0.0 & 0 & 0.0 \\
\hline Nausea & 1 & 3.0 & 0 & 0.0 & 0 & 0.0 & 0 & 0.0 \\
\hline Peptic ulcer perforation $\dagger$ & 1 & 3.0 & 0 & 0.0 & 1 & 33.3 & 0 & 0.0 \\
\hline Vomiting & 1 & 3.0 & 0 & 0.0 & 0 & 0.0 & 0 & 0.0 \\
\hline Infections and infestations & 6 & 18.2 & 1 & 16.7 & 0 & 0.0 & 0 & 0.0 \\
\hline Diverticulitisł & 1 & 3.0 & 0 & 0.0 & 0 & 0.0 & 0 & 0.0 \\
\hline Ear infection & 1 & 3.0 & 0 & 0.0 & 0 & 0.0 & 0 & 0.0 \\
\hline Influenza & 1 & 3.0 & 0 & 0.0 & 0 & 0.0 & 0 & 0.0 \\
\hline Lower respiratory tract infection $\dagger$ & 1 & 3.0 & 0 & 0.0 & 0 & 0.0 & 0 & 0.0 \\
\hline Pneumonia† & 1 & 3.0 & 1 & 16.7 & 0 & 0.0 & 0 & 0.0 \\
\hline General disorders and administration & 5 & 15.2 & 0 & 0.0 & 1 & 33.3 & 0 & 0.0 \\
\hline Injection site itching $\ddagger$ & 1 & 3.0 & 0 & 0.0 & 0 & 0.0 & 0 & 0.0 \\
\hline Injection site mass $\$$ & 1 & 3.0 & 0 & 0.0 & 0 & 0.0 & 0 & 0.0 \\
\hline Injection site pain $ћ$ & 1 & 3.0 & 0 & 0.0 & 0 & 0.0 & 0 & 0.0 \\
\hline Injection site reaction $\downarrow$ & 1 & 3.0 & 0 & 0.0 & 0 & 0.0 & 0 & 0.0 \\
\hline Sudden death $\dagger, \S$ & 1 & 3.0 & 0 & 0.0 & 1 & 33.3 & 0 & 0.0 \\
\hline Blood and lymphatic system disorders & 2 & 6.1 & 1 & 16.7 & 1 & 33.3 & 0 & 0.0 \\
\hline Anaemia $\dagger$ & 1 & 3.0 & 1 & 16.7 & 0 & 0.0 & 0 & 0.0 \\
\hline Thrombocytopenia† & 1 & 3.0 & 0 & 0.0 & 1 & 33.3 & 0 & 0.0 \\
\hline Respiratory, thoracic and mediastinal & 3 & 9.1 & 0 & 0.0 & 0 & 0.0 & 0 & 0.0 \\
\hline Dyspnoea & 1 & 3.0 & 0 & 0.0 & 0 & 0.0 & 0 & 0.0 \\
\hline Pleural effusion $\dagger$ & 1 & 3.0 & 0 & 0.0 & 0 & 0.0 & 0 & 0.0 \\
\hline Pleuritic pain & 1 & 3.0 & 0 & 0.0 & 0 & 0.0 & 0 & 0.0 \\
\hline Metabolism and nutrition disorders & 2 & 6.1 & 2 & 33.3 & 0 & 0.0 & 0 & 0.0 \\
\hline Hypoglycaemia & 1 & 3.0 & 1 & 16.7 & 0 & 0.0 & 0 & 0.0 \\
\hline Back pain & 1 & 3.0 & 1 & 16.7 & 0 & 0.0 & 0 & 0.0 \\
\hline Musculoskeletal pain & 1 & 3.0 & 0 & 0.0 & 0 & 0.0 & 0 & 0.0 \\
\hline Psychiatric disorders & 2 & 6.1 & 0 & 0.0 & 0 & 0.0 & 0 & 0.0 \\
\hline Confusional state $\dagger$ & 1 & 3.0 & 0 & 0.0 & 0 & 0.0 & 0 & 0.0 \\
\hline Disorientation & 1 & 3.0 & 0 & 0.0 & 0 & 0.0 & 0 & 0.0 \\
\hline Investigations & 1 & 3.0 & 0 & 0.0 & 0 & 0.0 & 0 & 0.0 \\
\hline Blood glucose increased & 1 & 3.0 & 0 & 0.0 & 0 & 0.0 & 0 & 0.0 \\
\hline Skin and subcutaneous tissue disorders & 1 & 3.0 & 0 & 0.0 & 0 & 0.0 & 0 & 0.0 \\
\hline Erythematous rash $\ddagger$ & 1 & 3.0 & 0 & 0.0 & 0 & 0.0 & 0 & 0.0 \\
\hline Vascular disorders & 1 & 3.0 & 0 & 0.0 & 0 & 0.0 & 0 & 0.0 \\
\hline Hypertensive crisis $\dagger$ & 1 & 3.0 & 0 & 0.0 & 0 & 0.0 & 0 & 0.0 \\
\hline
\end{tabular}

Using the Common Terminology Criteria for Adverse Events (CTCAE) version 5.0.

$\dagger$, Event considered as serious (Note: Only two events classified as 'diarrhoea' were considered as serious).

\#, Possibly related to study drug.

$\S$, Unrelated to study drug. 
had dose decreases (one for safety reasons and one for tumour burden reduction after surgery).

Nine treatments were discontinued and the leading cause was disease progression for four patients. The five other reasons were death (unrelated to treatment), reimbursement issue, toxicity, abdominal surgery and surgery.

\section{Safety end point}

\section{Adverse events or serious adverse events}

In all, $14(58.3 \%)$ patients out of 24 reported at least one adverse event (AE). A total of 44 AEs were reported but 11 of these were the same event in the same patient. In this case, the AEs were considered as a single event and the most severe grade was reported. Thus, this report is based on 33 AEs.

The AEs and SAEs are reported in Table 5. The most common ones were gastrointestinal disorders $(24.2 \%)$ followed by infections and infestations (18.2\%), general disorders and administration $(15.2 \%)$, respiratory, thoracic and mediastinal AEs $(9.1 \%)$, and blood and lymphatic system disorders. All the AEs from the different system organ classes were reported once except for diarrhoea, which was the most frequent one $(9.1 \%)$.

There were six (18.2\%) Grade 3 AEs and the most frequent ones were metabolism and nutritional disorders (33.3\%). Three (9.1\%) AEs were reported as Grade 4, the most frequent ones being blood and lymphatic system disorders, gastrointestinal disorders, general disorders and issues at administration. No Grade 5 AEs were reported.

Seven $(21.1 \%)$ treatment-related AEs were reported by six patients. The most frequent ones were general disorders and issues at administration (57.1\%). One AE $(3 \%)$, classified under gastrointestinal disorders, led to withdrawal from the study treatment, because of abdominal discomfort.

Fourteen serious AEs (SAEs) occurred in 7 (29\%) patients. The most frequent SAEs were gastrointestinal disorders and infections/infestations, reported three times each (25\%) (Table 5).

No SAEs were related to the treatment, considered symptomatic overdose with lanreotide nor related to pregnancy.

\section{Lab results}

All 24 patients had a baseline CgA result with a mean ( \pm s.d.) of $1154.9 \pm$ 1965.3. Sixteen $(66.7 \%)$ patients had a 3 -month result with a mean ( \pm s.d.) of $1027.1 \pm 1508.6$ and $14(58.3 \%)$ patients had a 6 -month result with a mean ( \pm s.d.) of $643.7 \pm 1059.9$.

The decrease in $\mathrm{CgA}$ level was significant between baseline and 3 months $(p=0.018)$ and between baseline and the 6-month follow-up visit $(p=0.0208)$. An increase in the CgA level was observed between the 3- and 6-month follow-up visits. However, this elevation was not statistically significant

\section{Discussion}

This study evaluated the level of satisfaction in diarrhoea and/or flushing control, the symptomatic response to treatment, the QoL, the drug exposure and the safety profile in patients treated with lanreotide Autogel over a 6-month period.

In all, $78.6 \%$ of the patients were satisfied with control of diarrhoea and all the patients reported improvement or stabilisation of flushing control when treated with lanreotide Autogel over a 6-month period. These results also imply that the improvement or stabilisation happens between 3 and 6 months of treatment.

The number of patients who had greater than $50 \%$ reduction in daily episodes of diarrhoea and flushing (as reported by patients) were $67 \%$ and $80 \%$, respectively, over a 6 -month period.

When assessing the Health-Related Quality of Life report, significant improvement was reported for Global Health Status, Endocrine Scale and the GI Symptom Scale. Numerical differences were noted in 10 out of the 12 outcome measures.

Furthermore, the treatment was generally well tolerated. The AEs and SAEs observed in the study were not frequent and similar to the ones observed with the somatostatin analogue treatment class. ${ }^{10}$

The positive findings provided by the study are aligned with previous studies. Fisher et al. ${ }^{11}$ conducted a 16-week doubleblind study followed by a 32-week open label phase to determine diarrhoea and flushing control in patients with NET who were treated with lanreotide Autogel. Patients receiving the treatment reported an improved diarrhoea and flushing control compared to those in the placebo group. This improvement was observed during the double-blind period and persisted in the open-label phase. In a 6-month open and non-controlled study, Ruszniewski et al. ${ }^{12}$ also found a significant decrease in flushing and diarrhoea by the end of the sixth month. Both studies also used QoL questionnaires to support the data.

\section{Limitations}

The small sample size in this study should be considered when interpreting the results from this registry as well as reported $p$-values. Patients were only included from the private sector sites in South Africa and cannot be generalised for the entire South African population. The demographics of patients in this study are not representative of the demographics of the South African population. 


\section{Acknowledgements Competing interests}

M.N., C.B. and A.M. are employees of Sanofi and fulfil roles within the Medical Affairs team. Other authors have previously received honoraria from Sanofi for speaking engagements and have received sponsorships to attend international congresses.

\section{Authors' contributions}

All authors contributed equally to this work.

\section{Funding information}

The research was funded by Sanofi South Africa.

\section{Data availability statement}

Data sharing is not applicable to the article as no new data were created or analysed in this study.

\section{Disclaimer}

The views and opinions expressed in this article are those of the authors and do not necessarily reflect the official policy or position of any affiliated agency of the authors.

\section{References}

1. Modlin IM, Oberg K, Chung DC, et al. Gastroenteropancreatic neuroendocrine tumours. Lancet Oncol. 2008;9(1):61-72.
2. Yadegarfar G, Friend L, Jones L, et al. Validation of the EORTC QLQ-GINET21 questionnaire for assessing quality of life of patients with gastrointestinal neuroendocrine tumours. Br J Cancer. 2013;108(2):301-310. https://doi.org/ neuroendocrine tumo

3. Caplin ME, Pavel M, Ćwikła JB, et al. Lanreotide in metastatic enteropancreatic neuroendocrine tumors. N Engl J Med. 2014;371(3):224-233. https://doi. org/10.1056/NEJMoa1316158

4. Khan S, Krenning EP, Van Essen M, Kam BL, Teunissen JJ, Kwekkeboom DJ. Quality of life in 265 patients with gastroenteropancreatic or bronchial neuroendocrine tumors treated with [177LuDOTA0,Tyr3]octreotate. J Nucl Med. 2011;52(9):13611368. https://doi.org/10.2967/jnumed.111.087932

5. WMA - The World Medical Association-WMA Declaration of Helsinki - Ethical principles for medical research involving human subjects [homepage on the Internet]. [cited 2019 Nov 4]. Available from: https://www.wma.net/policiespost/wmadeclaration-of-helsinki-ethical-principles-for-medical-researchinvolving-human-subjects/

6. Modlin IM, Pavel M, Kidd M, Gustafsson BI. Review article: Somatostatin analogues in the treatment of gastroenteropancreatic neuroendocrine (carcinoid) tumours. Aliment Pharmacol Ther. 2010;31(2):169-188. https://doi.org/10.1111/ j.1365-2036.2009.04174.x

7. Aaronson NK, Ahmedzai S, Bergman B, et al. The European Organization for research and treatment of cancer QLQ-C30: A quality-of-life instrument for use in international clinical trials in oncology. J Natl Cancer Inst. 1993;85(5):365-376. https://doi.org/10.1093/jnci/85.5.365

8. Martini C, Gamper E-M, Wintner L, et al. Systematic review reveals lack of quality in reporting health-related quality of life in patients with gastroenteropancreatic neuroendocrine tumours. Health Qual Life Outcomes. 2016;14(1):127. https:// neuroendocrine tumours. Health Qual
doi.org/10.1186/s12955-016-0527-2

9. Osoba D, Rodrigues G, Myles J, et al. Interpreting the significance of changes in health-related quality-of-life scores. J Clin Oncol. 1998;16(1):139-44. https://doi. org/10.1200/JCO.1998.16.1.139

10. Gomes-Porras M, Cárdenas-Salas J, Álvarez-Escolá C. Somatostatin analogs in clinical practice: A review. Int J Mol Sci. 2020;21(5):1682. https://doi.org/10.3390/ ijms21051682

11. Fisher GA, Wolin EM, Liyanage $\mathrm{N}$, et al. Patient-reported symptom control of diarrhea and flushing in patients with neuroendocrine tumors treated with lanreotide depot/autogel: Results from a randomized, placebo-controlled, double-blind and 32-week open-label study. Oncologist. 2018;23(1):16-24. https://doi.org/10.1634/theoncologist.2017-0284

12. Ruszniewski P, Ish-Shalom S, Wymenga $M$, et al. Rapid and sustained relief from the symptoms of carcinoid syndrome: Results from an open 6-month study of the 28-day prolonged-release formulation of Lanreotide. Neuroendocrinology. 2004; 80(4):244-251. https://doi.org/10.1159/000082875 